\title{
Tuning Promoter Strengths for Improved Synthesis and Function of Electron Conduits in Escherichia coli
}

\author{
Cheryl P. Goldbeck, ${ }^{\dagger}$ Heather M. Jensen, ${ }^{\ddagger}{ }^{\S}$ Michaela A. TerAvest, $"$ Nicole Beedle, ${ }^{\dagger}$ Yancey Appling, ${ }^{\dagger}$
} Matt Hepler, $\stackrel{\ddagger}{\ddagger}$ Guillaume Cambray, ${ }^{\perp, \#}$ Vivek Mutalik, ${ }^{\ddagger} \perp$ Largus T. Angenent, $"$ and Caroline M. Ajo-Franklin*,t,

Divisions of ${ }^{\dagger}$ Materials Sciences and ${ }^{\ddagger}$ Physical Biosciences, Lawrence Berkeley National Laboratory, Berkeley, California 94720 , United States

${ }^{\S}$ Department of Chemistry, University of California, Berkeley, California 94720, United States

"Department of Biological and Environmental Engineering, Cornell University, Ithaca, New York 14853, United States

${ }^{\perp}$ BIOFAB International Open Facility Advancing Biotechnology (BIOFAB), Emeryville, California 94608, United States

${ }^{\#}$ California Institute for Quantitative Biosciences, University of California, Berkeley, California, 94720, United States

\section{Supporting Information}

ABSTRACT: Introduction of the electron transfer complex MtrCAB from Shewanella oneidensis MR-1 into a heterologous host provides a modular and molecularly defined route for electrons to be transferred to an extracellular inorganic solid. However, an Escherichia coli strain expressing this pathway displayed limited control of MtrCAB expression and impaired cell growth. To overcome these limitations and to improve heterologous extracellular electron transfer, we used an E. coli host with a more tunable induction system and a panel of constitutive promoters to generate a library of strains that separately transcribe the $m t r$ and cytochrome $c$ maturation $(\mathrm{ccm})$ operons over 3 orders of magnitude. From this library, we identified strains that show 2.2 times higher levels of MtrC and MtrA and that have improved cell growth. We find that a 300-fold decrease in the efficiency of MtrC and MtrA synthesis with increasing $m t r$ promoter activity critically limits the maximum expression level of MtrC and MtrA. We also tested the extracellular electron transfer capabilities of a subset of the strains using a three-electrode microbial electrochemical system. Interestingly, the strain with improved cell growth and fewer morphological changes generated the largest maximal current per cfu, rather than the strain with more MtrC and MtrA. This strain also showed $\sim 30$-fold greater maximal current per cfu than its $\mathrm{ccm}$-only control strain. Thus, the conditions for optimal MtrCAB expression and anode reduction are distinct, and minimal perturbations to cell morphology are correlated with improved extracellular electron transfer in E. coli.

KEYWORDS: multiheme cytochrome c, extracellular electron transfer, cytochrome c biogenesis, secretion, membrane protein expression, Shewanella oneidensis MR-1

B th organisms and technological devices use electrons to carry information and energy. Creating a means for the bidirectional flow of electrons between living and nonliving systems would create new opportunities in energy conversion, ${ }^{1-3}$ biosensing, ${ }^{4}$ and biocomputing. ${ }^{5,6}$ However, engineering such a conduit involves overcoming the electrically insulating character of cellular membranes without significantly affecting cell viability. To make electronic connections to cells, most approaches rely on the introduction of diffusible redox species. $^{7-10}$ We recently established a novel synthetic biology approach to overcome this long-standing challenge by genetically introducing a heterologous extracellular electron transport pathway into Escherichia coli. ${ }^{11}$

This strategy takes advantage of the respiratory capability of the dissimilatory metal reducing bacterium Shewanella oneidensis MR-1. Under anaerobic conditions, this bacterium couples the oxidation of organic matter to the reduction of insoluble metals. Because insoluble metals cannot enter the cell, dissimilatory metal reducing bacteria use a transmembrane electron transfer pathway, which transports electrons from the cell interior to the extracellular surface. Genetic and biochemical studies have defined the pathway for anaerobic respiration of lactate with $\mathrm{Fe}$ (III) oxides or an anode serving as the terminal electron acceptor ${ }^{12}$ in S. oneidensis MR-1 (Figure 1a). L-Lactate dehydrogenase oxidizes L-lactate to pyruvate, ${ }^{13}$ and then pyruvate is converted to formate and acetate. ${ }^{14,15}$ Reducing equivalents resulting from L-lactate oxidation are delivered to the periplasmic reductase $\mathrm{CymA}^{16}$ via menaquinol. Electrons are transferred from CymA to $\mathrm{MtrA}^{17-19}$ and from MtrA to MtrC within the MtrCAB complex..$^{20,21}$ From MtrC,

Received: November 5, 2012

Published: January 14, 2013 


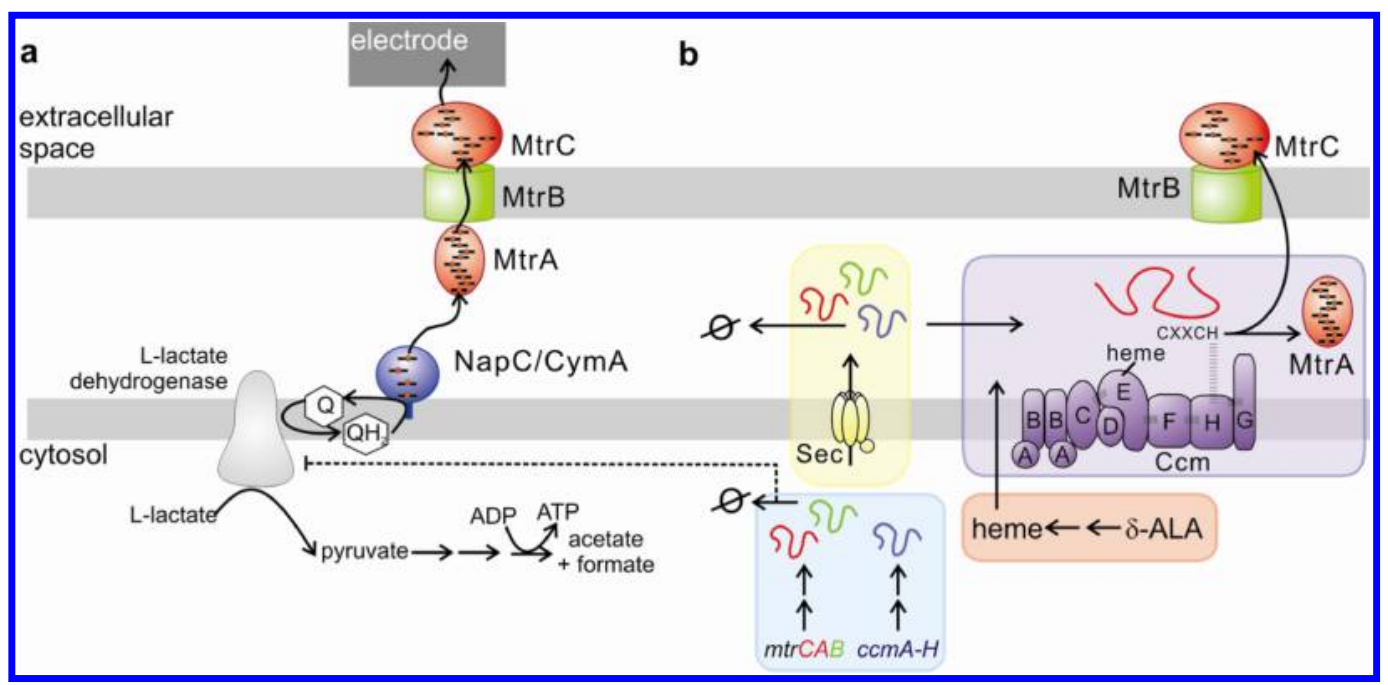

Figure 1. Multiple post-translational modifications are required to synthesize the components of the Mtr electron conduit, and misregulation of these processes has the potential to negatively impact extracellular respiration. (a) Route for electrons from lactate to an anode via the Mtr pathway. $\mathrm{L}$-Lactate is oxidized into pyruvate, and the resulting reducing equivalents are used to reduce menaquinone $(\mathrm{MQ})$ to menaquinol $\left(\mathrm{MQH} \mathrm{H}_{2}\right)$. In $S$. onedensis MR-1, CymA is the inner membrane cytochrome $c$ that accepts electrons from menaquinol; in E. coli, this function is achieved by NapC, as well as yet unidentified proteins. From the inner membrane protein, electrons are passed through the periplasm and outer membrane to extracellular electron acceptions via the MtrCAB complex. (b) After transcription and translation, the Mtr and Ccm polypeptides are secreted into the periplasm by the Sec secretion system. Excessive production of signal sequence-containing polypeptides can overload the Sec translocon, resulting in aggregation and/or retention of the polypeptides in the cytoplasm. The $\mathrm{Ccm}$ machinery then loads heme into the apocytochromes $c$ to form mature $\mathrm{MtrC}$ and MtrA. Low production of heme or inadequate concentrations of $\mathrm{Ccm}$ biogenesis machinery relative to the steady-state apocytochrome $c$ concentration can trigger degradation of the apocytochrome $c$ by periplasmic proteases such as DegP.

electrons can be transported to terminal electron acceptors through redox shuttles, ${ }^{22-27}$ direct contact, ${ }^{23,24}$ or possibly through other proteins on the cell surface (e.g., OmcA ${ }^{28}$ ). The electron conduit of this organism is also reversible and can accept electrons from a cathode to drive intracellular reduction reactions. $^{29}$

Previously, we transferred the MtrCAB electron transfer pathway into the model microbe $E$. coli and demonstrated that the resultant strain could reduce inorganic solids. ${ }^{11}$ In E. coli, $\mathrm{NapC}$ and other unidentified proteins partially substituted for the function of CymA in S. oneidensis MR-1. ${ }^{11}$ However, close examination of this strain, referred to here as the BL21(DE3) $m \operatorname{tr} C A B$ strain, reveals two key shortcomings: (i) this strain has dramatically slowed growth relative to its parent strain, and (ii) inducing transcription of the $m$ tr operon with isopropyl $\beta$-D-1thiogalactopyranoside (IPTG) caused the cell pellet to appear less red, ${ }^{11}$ suggesting that fewer $c$-type cytochromes were produced at higher transcription rates.

These challenges likely arise from the high degree of posttranslational modifications required to make c-type cytochromes (Figure $1 \mathrm{~b}$ ). MtrA is a $32-\mathrm{kD}$ periplasmic decaheme cytochrome $c{ }^{30}$ and $\mathrm{MtrC}$ is a $69-\mathrm{kD}$ cell-surface-exposed, ${ }^{31}$ lipid-anchored decaheme cytochrome $c .^{32} \mathrm{MtrC}$ requires $\mathrm{MtrB}^{33}$ a $72-\mathrm{kD}$ predicted twenty-eight strand $\beta$-barrel outer membrane protein, ${ }^{34}$ for correct assembly in the outer membrane $^{33}$ (Figure 1a). MtrA also appears to be necessary for MtrB stability. ${ }^{20,35}$ MtrC and MtrA biogenesis requires the cytochrome $c$ maturation $(\mathrm{ccm})$ genes, which encode the eight membrane proteins CcmABCDEFGH. Because transcription of the genomic $\mathrm{ccm}$ operon in $E$. coli is repressed under aerobic conditions, ${ }^{36}$ the $\mathrm{ccm}$ genes must be expressed from a plasmid to mature MtrC and MtrA. This is traditionally done with cotransformation of the helper plasmid, pEC86, which uses a tet promoter to constitutively express the E. coli $\mathrm{ccm}$ genes. ${ }^{37,38}$ After their synthesis, the $\mathrm{Ccm}$ and Mtr polypeptides are secreted into the periplasm (Figure $1 \mathrm{~b}$ ). The Ccm system plays a role in translocating heme into the periplasm and then catalyzes the formation of thioether bonds that link heme to two cysteine residues. ${ }^{39}$ Once these processes are complete, the axial ligands (typically histidine) are coordinated to the heme iron and the holocytochrome $c$ is folded ${ }^{39}$ (Figure $1 \mathrm{~b}$ ). Finally, MtrC and MtrB must be translocated to the outer membrane, presumably by type II secretion. ${ }^{40}$

This series of post-translational modifications creates multiple points for misregulation of the synthesis and maturation of the MtrCAB complex in E. coli. High levels of leader-sequence bearing polypeptides can saturate the signal recognition particle, leading to the misfolding of the unsecreted polypeptides and accumulation of cytoplasmic aggregates. ${ }^{41}$ This results in pleiotropic cellular impairments including an increase in the size and granularity of $E$. coli cells, decreased growth, and decreased expression of the inner membrane proteome. $^{42}$ Since L-lactate dehydrogenase is an inner membrane protein, secretion impairment has the potential to negatively impact the flux of electrons to menaquinone from lactate metabolism as well as the synthesis of MtrCAB (Figure 1). Misregulation of cytochrome $c$ synthesis can also be caused by inadequate heme, which will result in degradation of apocytochromes $c$ by the periplasmic proteases, such as $\mathrm{DegP}^{43}$ (Figure 1b). Thus c-type cytochrome biogenesis requires an appropriate balance between the rates and levels of polypeptide synthesis, heme biosynthesis, polypeptide secretion, and cytochrome $c$ maturation. Reflecting this, other groups have found that heterologous multiheme cytochrome $c$ expression in E. coli can be increased in two ways: (i) by enhancing heme biosynthesis with heme precursors, such as $\delta$-aminolevulinic acid $(\delta \text {-ALA })^{44}$ or rich media ${ }^{45}$ and (ii) by lowering apopolypeptide synthesis through weaker transcriptional promoters, ${ }^{46,47}$ different $E$. coli hosts, and lower copy number of the cytochrome $c$ genes. ${ }^{48}$ 
The slow growth of BL21(DE3) $m \operatorname{tr} C A B$ and decrease in cytochrome $c$ expression upon induction suggests that the basal transcription rate of $\mathrm{mtr}$ and the constitutive expression of $\mathrm{ccm}$ outpace the rates of heme synthesis, polypeptide secretion, and/or cytochrome $c$ maturation. Here, we hypothesize that by balancing the $\mathrm{ccm}$ and $\mathrm{mtr}$ transcription to avoid misregulation of post-transcriptional modifications, we can improve cytochrome $c$ expression, cell growth, and/or extracellular electron transfer relative to the BL21(DE3) $m \operatorname{trCAB}$ strain. Through the use of a more tightly regulated and tunable induction system and a panel of constitutive promoters, we created strains with varying expression of the $\mathrm{ccm}$ and $m \mathrm{tr}$ operons. We show that cytochrome $c$ expression and cell growth are strongly affected by the $m t r$ promoter activity. Surprisingly, we also find that the $\mathrm{ccm}$ and $\mathrm{mtr}$ promoter strengths for optimal cytochrome $c$ expression are distinct from those for improved anode reduction.

\section{RESULTS AND DISCUSSION}

Design of Strains with Variable Levels of Transcription of the $\mathrm{ccm}$ and $\mathrm{mtr}$ Operons. The overall goal of this work is to provide controllable and improved synthesis and function of the MtrCAB pathway in E. coli. The BL21(DE3) $m \operatorname{trCAB}$ strain contains a pET30a+-derived plasmid containing $m \operatorname{tr} C A B$ under the control of a $T 7$ lac promoter and a cytochrome $c$ maturation plasmid containing the $\mathrm{ccm}$ operon regulated by the constitutive tet promoter (pEC86). ${ }^{11}$ Designed as a control for the $c c m$ operon, the BL21(DE3) $\mathrm{ccm}$ strain only carries the pEC86 plasmid. Interestingly, the BL21(DE3) $m \operatorname{trCAB}$ and BL21(DE3) $\mathrm{ccm}$ strains grew $27 \pm 8 \%$ and $7 \pm 1 \%$ more slowly than the BL21(DE3) WT strain, respectively (Supporting Information Figure 1a). Moreover, the BL21(DE3) $m \operatorname{trCAB}$ strain shows 2fold greater forward scatter relative to BL21(DE3) WT strain (Supporting Information Figure $1 \mathrm{~b}$ ), indicating that MtrCAB expression causes an increase in cell size. Additionally, induced BL21(DE3) mtrCAB appear less red than uninduced cell pellets, ${ }^{11}$ indicating that increased transcription of $m \operatorname{tr} C A B$ led to decreased production of holocytochrome $c$.

Because expression of multiheme $c$-type cytochromes and membrane proteins have previously been improved by lowering transcriptional levels, ${ }^{49,50}$ we hypothesized that a similar strategy would improve control or levels of cytochrome $c$ expression or both. To achieve controlled expression, we changed the host strain to C43(DE3), which has lower basal and induced levels of T7 RNA polymerase relative to BL21(DE3) due to mutations in the lacUV5 promoter region. ${ }^{50}$ Using the methodology of Kelly et al., ${ }^{51}$ we calibrated the relative promoter activity by varying the concentration of IPTG. We found that induction of the T7 lac promoter can modulate transcription over $\sim 3$ orders of magnitude in the C43(DE3) strain (Supporting Information Figure $2 a-c$ and Supporting Information Table 1). We used this promoter (in the previously described $m \operatorname{tr} C A B$ plasmid $^{11}$ ) in the $C 43(\mathrm{DE} 3)$ strain to control transcriptional levels of $m \operatorname{tr} C A B$.

Since the BL21(DE3) $\mathrm{ccm}$ strain showed slower cell growth, which has been correlated with misregulation of membrane protein expression, ${ }^{41}$ we hypothesized that decreasing the level of $\mathrm{ccm}$ expression could improve cell growth and possibly functional expression of the MtrCAB pathway. Thus, we calibrated a series of constitutive promoters of varying strength generated by the BioFAB (Emeryville, CA; Mutalik et al., unpublished data), again using the methodology of Kelly et al. ${ }^{51}$
This library spanned 3.5 orders of magnitude in promoter strength (Supporting Information Figure $2 \mathrm{~d}-\mathrm{f}$ and Supporting Information Table 2), providing a wide window over which to tune $\mathrm{ccm}$ transcription. Golden Gate cloning ${ }^{52}$ was used to introduce the BioFAB promoters in front of the $\mathrm{ccm}$ operon, creating a library of plasmids with variable $\mathrm{ccm}$ transcription.

High Transcriptional Levels of $\mathrm{ccm}$ and $\mathrm{mtr}$ Decrease Cytochrome c Expression and Cell Growth. The $c c m$ plasmids were co-transformed with the previously described $m \operatorname{tr} C A B$ plasmid $^{11}$ into $\mathrm{C} 43(\mathrm{DE} 3)$ cells (Figure $2 \mathrm{a}$ ) to generate

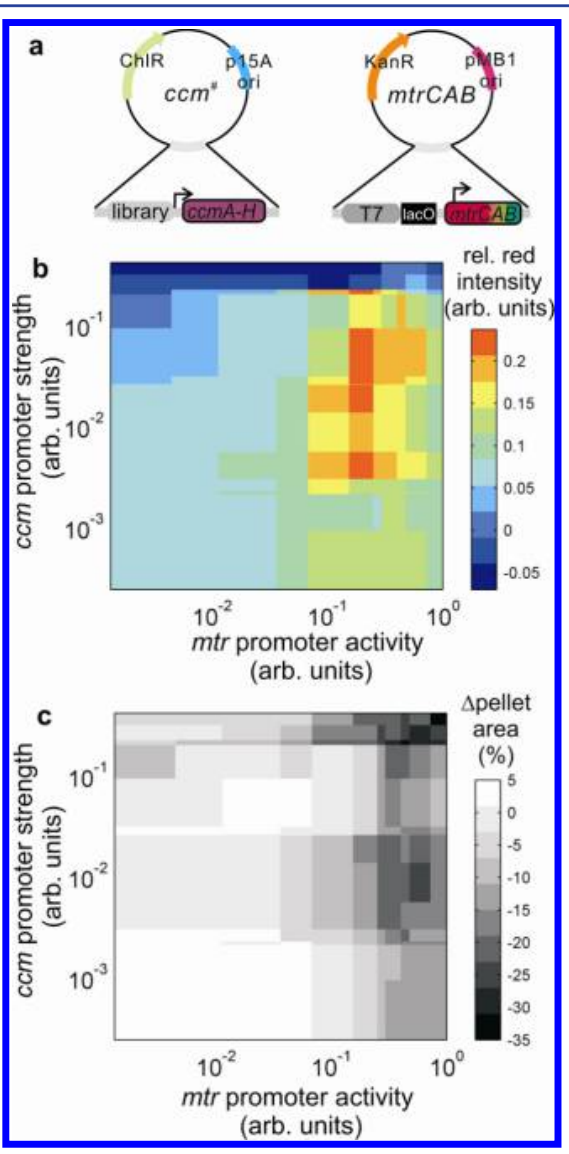

Figure 2. Cytochrome $c$ expression and cell growth are optimal in localized ranges of $\mathrm{mtr}$ promoter activity and $\mathrm{ccm}$ promoter strength. (a) Schematic of the $\mathrm{ccm}$ plasmid library and the $m \mathrm{tr} C A B$ plasmid used to create $\mathrm{ccm}^{\#} m \mathrm{trCAB}$ strains. (b) A heat map displaying the relative red intensity of the cell pellet of these strains as a function of the $m t r$ promoter activity and $\mathrm{ccm}$ promoter strength. On this scale, E. coli cells expressing no cytochrome $c$ and a purely red $E$. coli cell pellet would have relative red intensities of 0 and 2.1, respectively. This map reveals a localized region of maximal relative red intensity at intermediate $\mathrm{ccm}$ promoter strength and $m$ tr promoter activity. (c) A heat map displaying the change in apparent cell pellet size as a function of the $m t r$ promoter activity and $\mathrm{ccm}$ promoter strength shows sharply decreasing pellet size with increasing $m t r$ promoter activity. In both panels $b$ and $c$, the values plotted are the mean of at least three measurements, and granularity of each heatmap was set to the average of the standard deviation across all the measurements.

a series of $m \operatorname{tr} C A B$-expressing strains (Supporting Information Table 3). We notate the different transcriptional levels of $\mathrm{ccm}$ and $m t r$ operons in these cultures as $\mathrm{ccm}^{\#} m t r C A B^{\#}$ where the superscripts indicate the $\mathrm{ccm}$ promoter strength and $m t r$ promoter activity based on our calibrated scale in Supporting Information Tables 2 and 1, respectively. As a control, the 
C43(DE3) WT strain was generated by co-transformation of the empty pET30a+ and pACYC184 plasmids.

To screen the effects of simultaneously tuning the $\mathrm{ccm}$ promoter strength and the mtr promoter activity on cytochrome $c$ expression, the $c_{c m}^{\#} m t r C A B^{\#}$ strains were grown in 96-well plates with varying IPTG concentrations to generate 192 different transcriptional combinations. This number of combinations precluded direct analysis of cytochrome $c$ content. Instead, because $c$-type cytochromes are red in color, we quantified the relative red intensity of the cell pellets (calculated as described in Methods) and used this value as a metric for cytochrome $c$ production. To measure the relative red intensity, equal volumes of each culture were centrifuged, and images of the resulting cell pellets were acquired using a scanner. The heatmap shown in Figure $2 \mathrm{~b}$ summarizes the results of varying the transcriptional levels of both the $\mathrm{ccm}$ and $\mathrm{mtr}$ operons on the relative red intensity of the cell pellet. Unlike the BL21(DE3) mtrCAB strain, the $\mathrm{ccm}^{\#}$ $m \operatorname{tr} C A B^{\#}$ cultures show little or no relative red intensity in the absence of induction (Figure 2b, leftmost column). This increased control reflects the lower basal transcription of $\mathrm{T} 7$ RNA polymerase in the C43(DE3) strain. The pellets with the greatest relative red intensities were localized in the narrow range of $m$ tr promoter activities between $\sim 0.1$ to 0.3 arb. units and $\mathrm{ccm}$ promoter strengths between $\sim 0.01$ to $0.2 \mathrm{arb}$. units (Figure $2 \mathrm{~b}$ ). At $\mathrm{ccm}$ or $m \mathrm{tr}$ transcription levels outside this optimal range, the relative red intensity of the cell pellets decreased sharply. This decrease suggests that there is misregulation in the post-transcriptional processes. The $\mathrm{ccm}^{\#}$ $m+r C A B^{\#}$ culture with the greatest relative red intensity $(0.22 \pm$ 0.02 ) was more intensely red than the BL21(DE3) $m \operatorname{tr} C A B$ cells $(0.11 \pm 0.03)$, indicating that our screen produced a strain with higher expression levels of cytochrome $c$ than the original strain.

To assess the impact of $m t r$ and $c c m$ transcriptional levels on cell growth, we calculated (as described in Methods) the change in apparent cell pellet area relative to the C43(DE3) WT strain. This area is proportional to the pellet volume and thus the total cell biomass. As shown in the heatmap in Figure $2 c$, the apparent cell pellet area was unchanged in strains with both very low $m t r$ and $c \mathrm{~cm}$ transcriptional levels. The pellet area slightly decreased with increasing $\mathrm{ccm}$ promoter strength and steeply decreased with increasing mtr promoter activity, such that the apparent pellet area decreased by up to 35\% (Figure $2 c)$. Thus, transcription of the $m t r$ operon negatively impacts the maximal cell density. Moreover, cytochromes $c$ expression and cell growth were impacted in distinct manners by the transcriptional levels of the $\mathrm{ccm}$ and $m \mathrm{tr}$ operons.

Increasing the $\mathrm{ccm}$ Promoter Activity Results in Decreased Levels of $\mathrm{CcmE}$ and Cytochrome $c$ Maturation. The initial screen (Figure 2) suggested that $\mathrm{ccm}$ and $\mathrm{mtr}$ transcription levels impact cytochrome $c$ expression and total cell biomass, both of which are likely to be important for extracellular electron transfer in E. coli. To confirm these trends and to understand their molecular underpinnings, we determined the cytochrome $c$ abundance and cell density for a subset of cultures that showed high relative red intensities in the initial screen. These strains were grown as $50 \mathrm{~mL}$ cultures in flasks, and the final cell density was measured using traditional UV-vis spectroscopy. Diffuse reflectance spectroscopy was performed on intact cells to quantitatively determine [MtrC+MtrA $]$ per unit cell density using the unique absorption of $c$-type cytochromes at $552 \mathrm{~nm}$ (see Methods, Supporting
Methods, and Supporting Information Figure 3). Spectroscopic measurements (Supporting Information Figure 4a) and hemestaining of whole-cell lysates (Supporting Information Figure 4b) show that only MtrC and MtrA, rather than endogenous cytochromes $c$, contribute to the absorption at $552 \mathrm{~nm}$.

Measurements of relative red intensity and [MtrC+MtrA] from the same culture are highly correlated $(r=0.9$, Pearson's correlation coefficient, Supporting Information Figure 5a,b), confirming that relative red intensity is an effective screen for high cytochrome $c$ production per cell. As in the initial screening experiments, all the $c \mathrm{~cm}^{\#} m \mathrm{trCAB} B^{\#}$ strains measured have a low $[\mathrm{MtrC}+\mathrm{MtrA}]\left(2.5 \mu \mathrm{M} / \mathrm{OD}_{600 \mathrm{~nm}}^{\mathrm{DR}}\right)$ in the absence of induction by IPTG. The [MtrC+MtrA] increased to the 10-20 $\mu \mathrm{M} / \mathrm{OD}_{600 \mathrm{~nm}}^{\mathrm{DR}}$ range in the $\mathrm{ccm}^{\#} \mathrm{mtr}^{0.15}$ cultures (Figure $3 \mathrm{a}$ ).

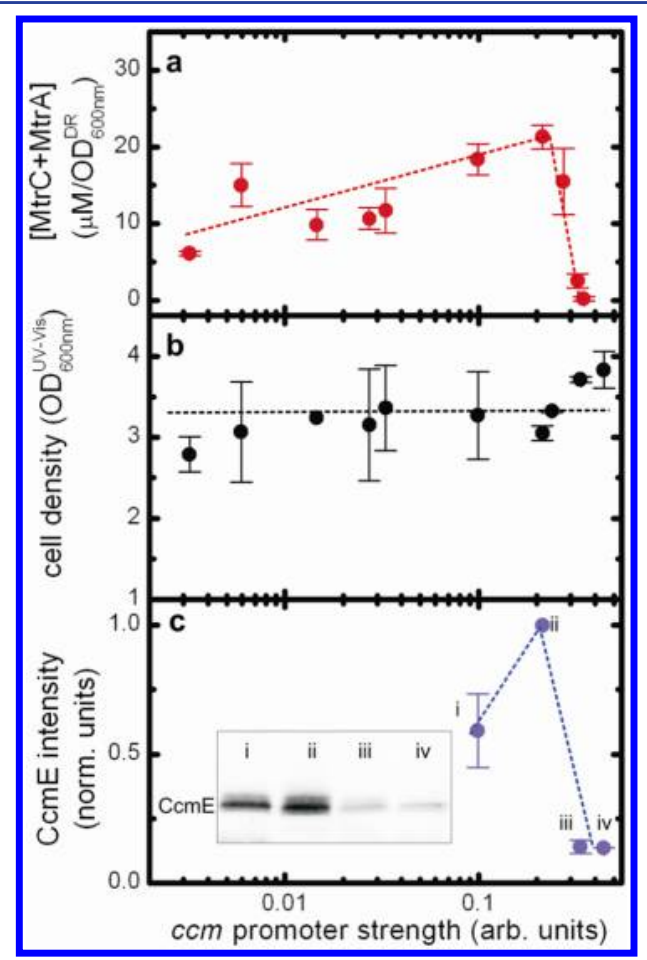

Figure 3. $c c m$ promoter strength modestly affects MtrC + MtrA concentration without affecting final cell density. (a) The total concentration of MtrA and MtrC per unit of $c \mathrm{~cm}^{\#} m \operatorname{mrCAB}{ }^{0.15}$ cultures as a function of $\mathrm{ccm}$ promoter strength. [MtrC+MtrA] reaches a maximum with $\mathrm{ccm}$ promoter strength of $\sim 0.1$ but is near background levels with $c c m$ promoter strengths $\geq 0.34$ arb. units. (b) The normalized $\mathrm{CcmE}$ intensity as a function of $\mathrm{ccm}$ promoter strength. The abundance of membrane-associated $\mathrm{CcmE}$ decreases with $\mathrm{ccm}$ promoter strengths $\geq 0.34 \mathrm{arb}$. units. The inset gel is the immunoblot of $\mathrm{CcmE}$ present in the membrane fraction of cell lysates from (i) $\mathrm{ccm}^{0.099} \mathrm{mtrCAB}^{0.15}$, (ii) $\mathrm{ccm}^{0.21} \mathrm{mtr} C A B^{0.15}$, (iii) $\mathrm{ccm}^{0.34} \mathrm{mtrCAB}{ }^{0.15}$, and (iv) $\mathrm{ccm}^{0.44} \mathrm{mtrCAB} B^{0.15}$ cultures. (c) The final cell density of $\mathrm{ccm}^{\#}$ $m \operatorname{tr} C A B^{0.15}$ cultures as a function of $\mathrm{ccm}$ promoter strength shows that $\mathrm{ccm}$ promoter strength does not significantly affect the cell density.

These observations confirm that the new C43(DE3)-derived strains offer enhanced control of decaheme cytochrome $c$ expression compared to the BL21(DE3) $m \operatorname{trCAB}$ strain. The [MtrC+MtrA] gradually increased by 3.5 times as the $\mathrm{ccm}$ promoter activity increased 67 -fold to 0.21 arb. units, but the [MtrC+MtrA] abruptly decreased with $\mathrm{ccm}$ promoter strengths above 0.34 arb. units (Figure $3 a$ ). These observed trends in the cytochrome $c$ abundance mimic the dependence of relative red intensity on $\mathrm{ccm}$ promoter strength found in the initial screen 


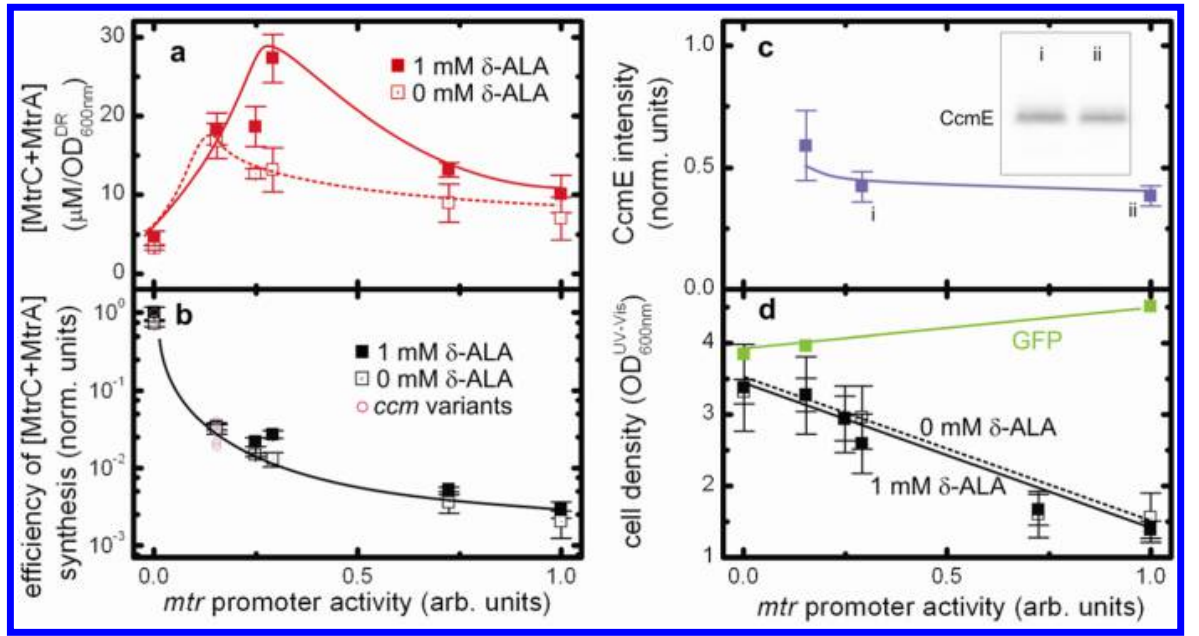

Figure 4. $m$ tr promoter activity sharply affects the efficiency of MtrC + MtrA synthesis and final cell density. (a) The total concentration of MtrA and MtrC per $\mathrm{OD}_{600 \mathrm{~nm}}^{\mathrm{DR}}$ of cells in $\mathrm{ccm}^{0.099} \mathrm{mtrCAB} B^{\#}$ cultures as a function of $m$ tr promoter activity in the presence of $0 \mathrm{mM} \delta$-ALA (filled squares) and $1 \mathrm{mM} \delta$-ALA(empty squares). The $[\mathrm{MtrC}+\mathrm{MtrA}]$ reaches a maximum with $m t r$ promoter activities of $\sim 0.2-0.25$ arb. units and sharply declines at higher promoter activities. (b) Efficiency of MtrC+MtrA synthesis as a function of $m \operatorname{tr}$ promoter activity for the $c \mathrm{~cm}^{0.099} \mathrm{mtrCAB}$ strain in the presence of $0 \mathrm{mM} \delta$-ALA (black filled squares) and $1 \mathrm{mM} \delta$-ALA (black empty squares) and the $c \mathrm{~cm}^{\#} m \operatorname{mrCAB}$ strains in the presence of $1 \mathrm{mM} \delta$ ALA (empty magenta circles). All cultures experience a dramatic decrease in the efficiency of cytochrome $c$ production with increasing $m t r$ promoter strength. (c) The normalized CcmE intensity as a function of $m t r$ promoter activities, showing that there is no significant decrease in the membraneassociated $\mathrm{CcmE}$ levels with increasing $m t r$ promoter activity. The inset gel is the immunoblot of CcmE present in the membrane fraction in (i) $\mathrm{ccm}^{0.099} \mathrm{mtrCAB} B^{0.29}$ and (ii) $\mathrm{ccm}^{0.099} \mathrm{mtrCAB} B^{1.0}$ cultures. (d). The final cell density as a function of $m t r$ promoter strength in the presence of $1 \mathrm{mM} \delta$ ALA (black filled circles) and absence of $\delta$-ALA (dotted black circles), showing a strong decrease in cell density with $m t r$ promoter activity $(r=$ -0.97 , Pearson's correlation coefficient). In contrast, the final cell density of a C43(DE3) strain harboring the pET30a+ GFP plasmid (filled green squares) increases slightly with IPTG induction.

(Supporting Information Figure 5c) and confirm that the decaheme cytochrome $c$ concentration per cell is nonmonotonically dependent on the $\mathrm{ccm}$ promoter activity. Also mirroring observations from our initial screen, the final cell density for $\mathrm{ccm}^{\#} \mathrm{mtrCAB} B^{0.15}$ cultures was high and independent of the $\mathrm{ccm}$ promoter strength (Figure $3 \mathrm{~b}$ ). These observations indicate that increasing the $\mathrm{ccm}$ promoter strength up to 0.21 arb. units modestly improves cytochrome $c$ expression without affecting cell growth.

We sought to explain the decrease in holocytochrome $c$ with high $\mathrm{ccm}$ promoter strengths $(\geq 0.34$ arb. units) to develop a deeper mechanistic understanding of the controls on decaheme cytochrome $c$ production. We hypothesized that high $\mathrm{ccm}$ promoter strengths could reduce the translation ${ }^{53}$ or secretion efficiency, ${ }^{41}$ which would decrease functional $\mathrm{Ccm}$ machinery present in the inner membrane and decrease cytochrome $c$ maturation. To quantitatively compare the membrane-associated CcmE levels across different strains (Figure 3c, inset), we normalized the $\mathrm{CcmE}$ band intensity of each strain by the band intensity arising from an equal mass of $\mathrm{ccm}^{0.21} \mathrm{mtrCAB} B^{0.15}$ culture lysate. This normalized $\mathrm{CcmE}$ intensity was linear with $\mathrm{CcmE}$ abundance over the range of our measurements (Supporting Information Figure 6a,b). The membraneassociated $\mathrm{CcmE}$ levels dramatically decreased with $\mathrm{ccm}$ promoter strengths $\geq 0.34$ arb. units, the same $\mathrm{ccm}$ promoter strengths that showed a sharp decline in $[\mathrm{MtrC}+\mathrm{MtrA}]$. Thus, this trend is consistent with a translation or secretion defect at high $\mathrm{ccm}$ promoter strengths, which decreases $\mathrm{Ccm}$ protein levels in the membrane. In turn, the low level of $\mathrm{Ccm}$ biogenesis machinery decreases the maturation of apo-proteins into holo-MtrC and holo-MtrA.

Efficiency of MtrC and MtrA Synthesis and Cell Growth Decrease Strongly with Increasing mtr Promoter Activity. We next investigated the effect of modulating the $m t r$ promoter activity on decaheme cytochrome $c$ expression and cell growth. We probed these trends as described above in the $\mathrm{ccm}^{0.099} m \operatorname{mrCAB} B^{\#}$ cultures because these cultures showed a robust expression of $c$-type cytochromes across a wide range of $m t r$ promoter activities in our initial screen (Figure 2b). As shown in Figure 4a, the $[\mathrm{MtrC}+\mathrm{MtrA}]$ in this strain sharply increased with increasing $m t r$ transcriptional levels, reaching a maximum when the $m t r$ promoter activity was 0.29 arb. units. Notably, the MtrC and MtrA abundance in the $c m^{0.099} m t r C A B^{0.29}$ culture is the highest that we have measured $\left(22 \pm 3 \mu \mathrm{M} / \mathrm{OD}_{600 \mathrm{~nm}}^{\mathrm{DR}}\right)$ and is 2.2 times higher than in the previously reported BL21(DE3) $m \operatorname{tr} C A B$ strain. After this optimum, the cytochrome $c$ concentration decreased to approximately half its maximal value.

We sought to understand the origin of this decrease to improve our mechanistic understanding of the controls on decaheme cytochrome $c$ production. We calculated the efficiency of cytochrome $c$ synthesis to assess how transcriptional levels of $\mathrm{mtr}$ are translated into holo-MtrC and holoMtrA levels. The efficiency synthesis was calculated by dividing the $[\mathrm{MtrC}+\mathrm{MtrA}]$ by the $m$ tr promoter activity and normalized such that the maximum efficiency was 1 . Strikingly, the efficiency of decaheme cytochrome $c$ synthesis in the $\mathrm{ccm}^{0.099}$ $m \operatorname{tr} C A B$ strain (solid black squares) drops by $\sim 300$-fold as the $m t r$ promoter activity is increased (Figure $4 \mathrm{~b}$ ). The same analysis of the $\mathrm{ccm}^{\#} m t r C A B^{0.001}$ and $\mathrm{ccm}^{\#} m t r C A B^{0.15}$ cultures show that different $c c m$ transcriptional levels (empty magenta circles) have a minor impact on the efficiency of cytochrome $c$ synthesis, as long as the transcriptional levels are below those that result in secretion impairment. Thus, at high $m$ tr promoter activities, the increase in $m t r$ transcripts is negated by the lower efficiency of synthesis from these transcripts, leading to a decreased yield of decaheme cytochrome $c$. 


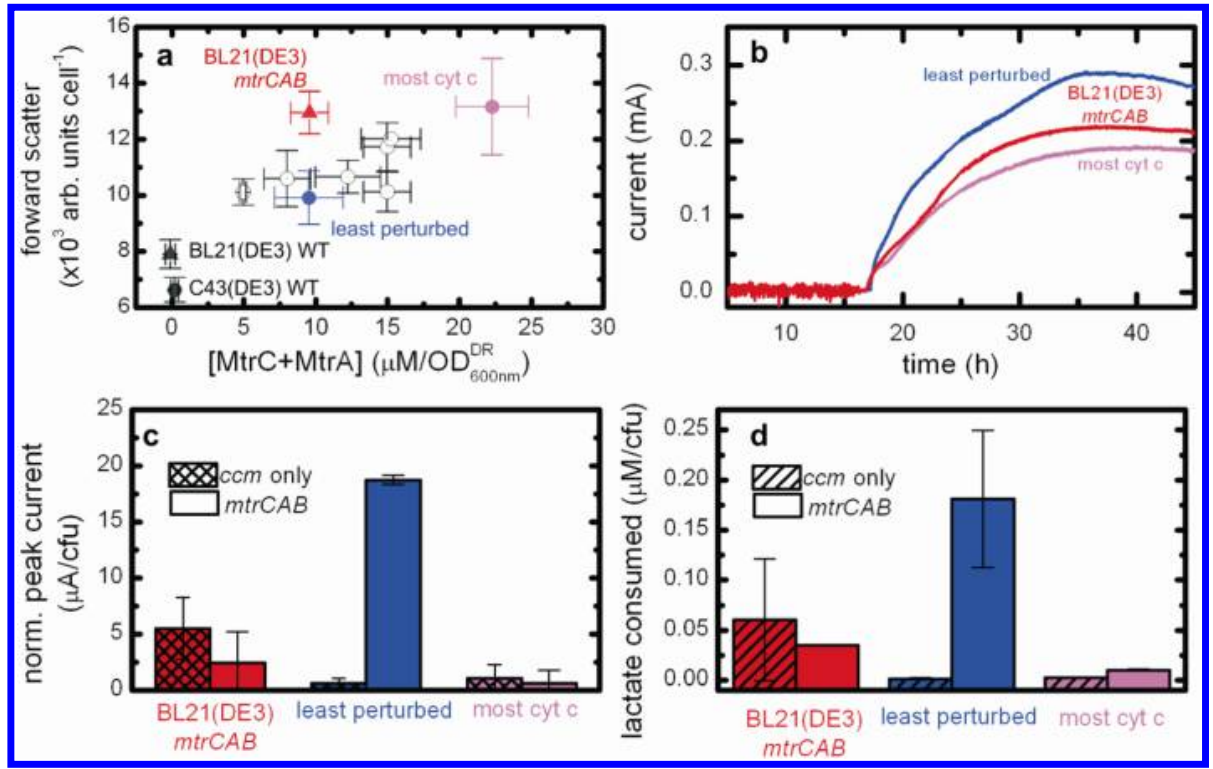

Figure 5. A C43-derived $m \operatorname{tr} C A B$ strain with a less perturbed cellular morphology can transfer electrons to an anode. (a) Forward scatter versus $[\mathrm{MtrC}+\mathrm{MtrA}]$ for $\mathrm{ccm}^{\#} m \mathrm{trCAB}{ }^{\#}$ cultures. Highlighted are the BL21(DE3) WT (black triangle), C43(DE3) WT (black circle), BL21(DE3) mtrCAB (red triangle), least perturbed (blue circle), and most cytochrome $c$ (magenta circle) strains. (b) Representative electrochemical measurements of BL21(DE3) $m \operatorname{trCAB}$ culture (red line), the least perturbed culture (blue line), and most cytochrome $c$ culture (magenta line). (c) Maximal current per cfu for the BL21(DE3) mtrCAB cells (solid red bar), the least perturbed culture (solid blue bar), and most cytochrome $c$ culture (solid magenta bar) and their respective ccm-only strains: the BL21(DE3) ccm (cross-hashed red bar), least perturbed ccm (cross-hashed blue bar), and most cytochrome $c \mathrm{ccm}$ (cross-hashed magenta bar). There is a significant difference in maximal current per cfu between the least perturbed culture and its control, the least perturbed $c \mathrm{~cm}$ strain ( $p$-value $=0.01$, Student's one-tailed $t$ test with equal variances). (d) Lactate consumption per cfu for the BL21(DE3) mtrCAB cells (solid red bar), the least perturbed (solid blue bar), and most cytochrome $c$ (solid magenta bar) cultures and their respective $\mathrm{ccm}$-only strains $\mathrm{BL21}(\mathrm{DE} 3) \mathrm{ccm}$ (hashed red bar), least perturbed $\mathrm{ccm}$ (hashed blue bar), and most cytochrome $c \mathrm{ccm}$ (hashed magenta bar). There is a significant difference in lactate consumed per cfu between the least perturbed and least perturbed $\mathrm{ccm}$ strains $(p$-value $=0.03$, Student's one-tailed $t$ test with equal variances).

Two known mechanisms could result in decreased efficiency of cytochrome $c$ synthesis with increasing cytochrome $c$ transcript levels: (i) saturation of translation or secretion ${ }^{41}$ by high $m t r$ expression ${ }^{53}$ could decrease $\mathrm{Ccm}$ and thus decreased ability to mature protein, ${ }^{43}$ or (ii) inadequate heme production $^{43}$ could lead to increased degradation of partially matured decaheme cytochrome $c$. We first probed membraneassociated $\mathrm{CcmE}$ abundance as a function of $\mathrm{mtr}$ promoter activity (Figure 4c). The membrane-associated CcmE levels were within 1 standard deviation of each while the $m t r$ promoter activity was varied by $\sim 5$-fold, indicating that there is no significant decrease in the $\mathrm{Ccm}$ biogenesis machinery of the strain (Figure 4c). Next, spent media from highly induced $\mathrm{ccm}^{0.099} \mathrm{mtrCAB} B^{0.72}$ cultures supplemented with the heme biosynthesis precursor $\delta$-ALA was analyzed spectroscopically for heme. The visibly red media contained $\sim 0.3 \mu \mathrm{M}$ heme $\mathrm{b}$ (not shown), ruling out heme limitation as a cause of the decrease in cytochrome $c$ synthesis efficiency. Further supporting this idea, we measured the $[\mathrm{MtrC}+\mathrm{MtrA}]$ in $\mathrm{ccm}^{0.099}$ mtrCAB cultures lacking $\delta$-ALA (dotted red squares, Figure $4 \mathrm{a}$ ). While diminished bioavailability of heme decreases the efficiency of holocytochrome $c$ synthesis by $\sim 30 \%$ across all $m$ tr promoter activities, it does not significantly alter the relationship between efficiency of cytochrome $c$ synthesis and mtr promoter activity (Figure $4 \mathrm{~b}$ ). Thus, our data rule out decreased $\mathrm{Ccm}$ biogenesis machinery and inadequate heme production as causes for the stark decrease in efficiency of cytochrome $c$ synthesis with increasing $m$ tr promoter activity.

To probe the impact of the $m t r$ promoter on cell growth, we measured the final cell density in the $\mathrm{ccm}^{0.099} \mathrm{mtr} C A B^{\#}$ cultures.
We observed that the cell density is negatively correlated with increasing $m$ tr promoter activity in the presence (solid black squares) or absence (hollow black squares) of $\delta$-ALA $(r=$ -0.97 , Pearson's correlation coefficient, Figure 4d). As described above, neither polypeptide synthesis nor heme bioavailability are limited in this strain. Therefore, the decrease in growth could be caused by toxicity of IPTG, MtrC, MtrA, or MtrB. To determine whether the decrease in cell density was caused by IPTG, we measured the final cell density of a C43(DE3) strain expressing GFP from the T7 lac promoter induced with varying [IPTG]. The final cell density of these cultures increased slightly with IPTG (solid green squares, Figure 4d), indicating that IPTG does not negatively influence growth. Additionally, comparison of the cytochrome $c$ abundance data in Figure $4 \mathrm{a}$ and $4 \mathrm{~d}$ shows that [MtrC $+\mathrm{MtrA}]$ is not strongly correlated with cell density $(r=-0.28$, Pearson's correlation coefficient). For example, the $\mathrm{ccm}^{0.099}$ $m \operatorname{trCAB} B^{0.24}$ and $\mathrm{ccm}^{0.099} \mathrm{mtrCAB} B^{1.0}$ cultures have similar [MtrC + MtrA] (Figure 4a), yet the $c m^{0.099} m t r C A B^{1.0}$ cultures have a final cell density that is $50 \%$ of the $\mathrm{ccm}^{0.099} \mathrm{mtrCAB} B^{0.24}$ cultures (Figure $4 \mathrm{~d}$ ). Therefore, our data suggest that the decrease in cell density with $m$ tr promoter activity is not due to toxicity of IPTG, MtrA, or MtrC.

Anode-Reduction Correlates with Minimal Alterations in Cell Morphology and Moderate MtrC and MtrA Expression. Our central hypothesis is that the number of $\mathrm{MtrCAB}$ electron conduits as well as minimal perturbations to cell growth and physiology may be important to achieve extracellular electron transfer in E. coli. From the assays and screens above, we selected several $\mathrm{ccm}^{\#} m t r C A B^{\#}$ cultures with 
high cytochrome $c$ levels $\left([\mathrm{MtrC}+\mathrm{MtrA}] \geq 10 \mu \mathrm{M} / \mathrm{OD}_{600 \mathrm{~nm}}^{\mathrm{DR}}\right)$ and high final cell density (cell density $\geq 2 \mathrm{OD}_{600 \mathrm{~nm}}^{\mathrm{UV}-\mathrm{vis}}$ ). To provide an additional metric of overall cell morphology, we examined the forward scatter of these strains by flow cytometry (Figure 5a). Just as cells from the BL21(DE3) mtrCAB strain (Figure 5a, red filled triangle) showed increased scatter relative to the BL21(DE3) WT strain (Figure 5a, black filled triangle), cells from all of the $\mathrm{ccm}^{\#} m t r C A B^{\#}$ cultures (Figure 5a, circles) also showed increased forward scatter compared to the C43(DE3) WT strain (Figure 5a, black filled circle). These measurements indicate expression of MtrCAB causes significant morphological changes without substantially altering the final cell density.

We sought to understand the correlation of cellular cytochrome $c$ levels and cellular morphology to the ability of cells to transfer electrons to an electrode. Using a comparison of forward scatter versus [MtrC+MtrA] (Figure 5a), we selected a culture that shows less morphological perturbations (solid blue circle, $\mathrm{ccm}^{0.033} \mathrm{mtrCAB} B^{0.15}$, henceforth referred to as the "least perturbed" culture) and a culture that shows higher [MtrC+MtrA] (solid magneta circle, $\mathrm{ccm}^{0.099} \mathrm{mtrCAB}{ }^{0.29}$, henceforth referred to as the "most cytochrome $c$ " culture), relative to the original BL21(DE3) strain. These strains and their respective $\mathrm{ccm}$-only controls were inoculated at a high cell density into a three-electrode microbial electrochemical system. $^{54}$ The working electrode was poised at $0.2 \mathrm{~V}$ versus $\mathrm{Ag} / \mathrm{AgCl}$ (satd $\mathrm{KCl})$, L-lactate was supplied as the electron donor, and the electric current production was recorded over several days (Figure 5b). Interestingly, the least perturbed strain achieved higher current than either the BL21(DE3) $m \operatorname{tr} C A B$ strain or most cytochrome $c$ strain. Additionally, the least perturbed strain also produced $\sim 30$-fold greater maximum current per $\mathrm{cfu}$ than its counterpart $\mathrm{ccm}$-only strain (significantly different with a $p$-value $=0.01$, Figure $5 \mathrm{c}$ ), indicating that reduction of the electrode in the least perturbed strain is enhanced by the presence of the $\mathrm{MtrCAB}$ electron conduit. In contrast, both the BL21(DE3) mtrCAB and most cytochrome $c$ strains showed low maximal current per cfu, which were not statistically significant from their respective $c c m$-only strains ( $p$-value $=0.25$, Figure 5c). Although $S$. oneidensis MR-1 typically produces $\sim 1000$ times higher current than the least perturbed strain in these three-electrode electrochemical systems, the difference in current per cfu between the least perturbed strain in comparison to the other E. coli strains indicates it is possible to engineer anode reduction into new organisms using the MtrCAB electron conduit.

Additionally, HPLC analysis of the spent media (Figure 5d) shows that the most lactate per cfu was consumed by the least perturbed culture, supporting the hypothesis that electrons delivered to the electrode originate from E. coli metabolism of L-lactate. These observations indicate that heterologous expression of MtrCAB allows E. coli to transfer metabolic electrons to an electrode and that phenotypic features and $[\mathrm{MtrC}+\mathrm{MtrA}]$ are correlated with the efficiency and rate of extracellular electron transfer.

Transcriptional Balance Is Critical for Optimal in Vivo Synthesis and Function of Electron Conduits. Using our library of transcriptional variants, we are able to quantitatively define regimes in which different mechanisms limit further synthesis of MtrC and MtrA (Figure 6). Below a threshold level of $\mathrm{ccm}$ transcription, decaheme cytochrome $c$ is not produced because insufficient $\mathrm{Ccm}$ complexes are available to mature the

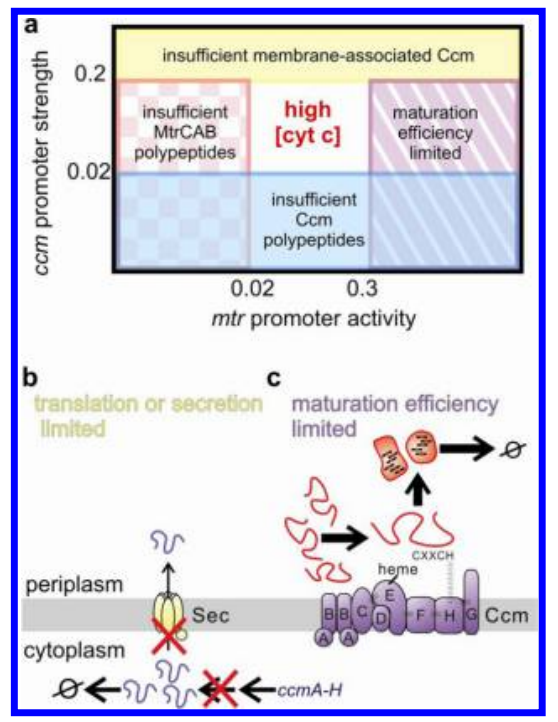

Figure 6. Constraints on decaheme cytochrome $c$ production. (a) Phase diagram showing regions of limited and maximal MtrC and MtrA expression in relation to $\mathrm{ccm}$ promoter strength and $m t r$ promoter activity. (b) Schematic illustrating that lowered cytochrome $c$ expression at high $\mathrm{ccm}$ promoter strength is due to a decrease in the $\mathrm{Ccm}$ complex abundance, which prevents maturation of cytochromes c. (c) One proposed mechanism that would account for decreased cytochrome maturation at high $m t r$ promoter activity. Poor processivity of the E. coli $\mathrm{Ccm}$ machinery coupled with a high concentration of apocytochromes $c$ results in incompletely matured MtrC and MtrA, which are subsequently degraded by periplasmic proteases.

apoproteins (Figure 6a). Above a certain limit, increasing the $\mathrm{ccm}$ transcriptional level actually decreases the membraneassociated $\mathrm{Ccm}$ biogenesis machinery (Figure 6b), setting a maximum level of $\mathrm{Ccm}$ biogenesis machinery available to mature apocytochrome $c$. Most critically, holocytochrome $c$ synthesis was sharply dependent on the $m t r$ promoter activity. Increased $m t r$ transcription (Figure 6a) is outweighed by the decreased efficiency of MtrC and MtrA synthesis, resulting in decreased decaheme cytochrome $c$ abundance.

To our knowledge, this is the first report systematically documenting a decrease in multiheme cytochrome $c$ production efficiency, although other groups have shown that low induction levels are optimal for heterologous expression of multiheme cytochrome $c$ in E. coli. ${ }^{46-48}$ Neither low heme abundance nor impairment in secretion account for this drop in efficiency of cytochrome $c$ production. While it is possible that increasing levels of MtrC inhibit its correct localization into the outer membrane, previous work in E. coli ${ }^{40}$ suggests that mislocalization of MtrC is unlikely to affect the overall levels of decaheme cytochrome $c$. We hypothesize that the low efficiency is due to an as-yet uncharacterized mechanism. One possible scenario is that the $\mathrm{Ccm}$ complex is insufficiently processive to rapidly mature decaheme cytochromes $c$ (Figure $6 \mathrm{c}$ ). Thus, the apoprotein level increases, $\mathrm{CXXCH}$ motifs from different polypeptides compete for binding to $\mathrm{Ccm}$ complex, resulting in a greater fraction of apoproteins being only partially matured and degraded. Three observations lend support to this idea: (i) heterologous expression of single heme cytochromes $c$ does not show the same dramatic dependence on transcriptional levels, ${ }^{55}$ (ii) the modest 3.5 -fold improvement of cytochrome $c$ production with 67 -fold increase in $\mathrm{ccm}$ transcriptional levels suggests that the $\mathrm{Ccm}$ complex is an inefficient enzyme for 
decaheme cytochrome $c$ maturation (Figure 6a), and (iii) in $S$. oneidensis MR-1 $40 \%$ of MtrC polypeptides never mature, suggesting that decaheme cytochrome $c$ maturation is intrinsically inefficient. ${ }^{56}$

In addition to the complexity of decaheme cytochrome $c$ synthesis, the function of MtrCAB electron conduits in E. coli is not straightforward. Extracellular electron transfer in E. coli not only is governed by the cellular concentration of decaheme cytochrome $c$ but also is correlated with minimal perturbations to cellular growth and morphology. Both MtrCAB subsystemspecific and more cellular-wide properties could underlie this observation. For example, a difference in ratio of MtrA:MtrB:MtrC or a change in percentage of properly localized MtrC, MtrB, or MtrA could negatively impact extracellular respiration without changing the decaheme content per cell. Alternatively, more cell-wide perturbations may be at root of this. For example, under aerobic growth conditions, the membrane stress response has been shown to decrease the rate of $\mathrm{O}_{2}(\mathrm{~g})$ consumption and impact the inner membrane proteome. ${ }^{41,50}$ Since reducing equivalents from Llactate must pass through the inner membrane proteins Llactate dehydrogenase and $\mathrm{NapC}$, these alterations could critically affect the availability of electrons to enter the MtrCAB electron conduit. Thus, future investigations will attempt to untangle these many possibilities to more firmly understand the factors critical for extracellular respiration in E. coli. Indeed, given the degree to which this system interfaces with host machinery, we anticipate that additional systems-level analysis will improve heterologous extracellular respiration to levels practical for biosensing and bioenergy applications.

Conclusions. Here we report the impact of tuning $\mathrm{ccm}$ and $m t r$ transcription on the synthesis and function of the MtrCAB electron conduit in E. coli. The use of the C43(DE3) strain enables the synthesis of MtrCAB to be controlled and improves MtrC and MtrA expression by 2.2 -fold. Surprisingly, the strain that produces the greatest current at the anode does not have the greatest abundance of MtrA and MtrC. Instead, it has a moderate amount of decaheme cytochromes $c$ and minimal perturbations in its cellular morphology. This strain delivers metabolic electrons to an electrode at a rate $\sim 30$-fold faster than $\mathrm{ccm}$-only controls. While the efficiency of this process is modest in comparison to naturally occurring dissimilatory metal-reducing bacteria, this work indicates that it is possible to genetically engineer molecularly defined extracellular electron transfer into an organism.

\section{METHODS}

Additional details may be found in the Supporting Information.

Plasmids, Strains, and Growth Conditions. The strains used in this study are listed in Supporting Information Table 3. The BL21(DE3) WT, BL21(DE3) $c \mathrm{~cm}$, and BL21(DE3) $m \operatorname{tr} C A B$ strains were described previously. ${ }^{11}$ The pFAB\#-GFP plasmids were obtained from the BioFAB (Emeryville, CA). To create a library of plasmids containing different promoters in front of the $\mathrm{ccm}$ coding sequence, Golden Gate cloning ${ }^{52}$ was used to replace the tet promoter in $\mathrm{pEC} 86^{37,38}$ with BioFABderived promoter sequences.

All strains were grown in $2 \mathrm{xYT}$ media with $50 \mu \mathrm{g} \mathrm{mL}$ kanamycin and $30 \mu \mathrm{g} \mathrm{mL} \mathrm{m}^{-1}$ chloramphenicol. Growth of cultures in 96-deep-well plates was done at $37^{\circ} \mathrm{C}$ with 275 -rpm shaking, and growth in $250 \mathrm{~mL}$ flasks was done at $30{ }^{\circ} \mathrm{C}$ with 250 -rpm shaking. Transcription of $m \operatorname{tr} C A B$ was induced by addition of IPTG when the cultures reached an $\mathrm{OD}_{600 \mathrm{~nm}}^{\mathrm{UV} \text { nis }}$ of 0.5 (typically 2-3 h after inoculation). Cultures were grown for a total of $20 \mathrm{~h}$ after inoculation.

Analysis of Cell Pellets for Assessment of Cytochrome c Expression and Cell Growth. After growth in 96-well plates, $150 \mu \mathrm{L}$ of culture was pelleted in clear, V-bottom 96-well polystyrene plates (ThermoScientific, Waltham, MA). The plates containing the cell pellets were scanned using a Scanjet G4050 scanner (HP, Palo Alto, CA). Regions of interest from each pellet were identified using the Matlab (R2009b) Image Processing Toolbox (MathWorks, Natick, MA). The relative red intensity was calculated by taking the ratio intensity in the red channel $\left(I_{\text {red }}\right)$ to the grayscale intensity $\left(I_{\text {gray }}\right)$ and subtracting the red to gray ratio of $\mathrm{C} 43(\mathrm{DE} 3) \mathrm{WT}$ cells: relative red intensity $=\left(I_{\text {red }}\right) /\left(I_{\text {gray }}\right)-\left(I_{\text {red }}^{C 43(D E 3) W T}\right) /$ $\left(I_{\text {gray }}^{C 43(D E 3) W T}\right)$. To calculate the change in cell pellet area, the difference in area between C43(DE3) WT cell pellet and the area of the cell pellet of interest was divided by the C43(DE3) WT cell pellet area.

Quantitative Assessment of Cytochrome c per Cell Density by Diffuse Reflectance Spectroscopy. Cell suspensions of $\mathrm{OD}_{600 \mathrm{~nm}}^{\mathrm{UV}-\mathrm{vis}} \sim 3-4 \mathrm{OD}$ were reduced by sodium dithionite at a final concentration of $10 \mathrm{mM}$, and $\sim 400 \mathrm{uL}$ was loaded into a transparent chamber consisting of $0.08 \mathrm{~cm}$ deep, $22 \mathrm{~mm}$ square Secure-Seal hybridization gaskets (Life Technologies, Eugene, OR) adhered to $25 \mathrm{~mm}$ wide square glass coverslip. The absorbance spectrum of the cell suspension was measured using Quality Spec Pro UV-vis spectrometer equipped with a Muglight diffuse reflectance attachment (ASD Inc., Boulder, $\mathrm{CO}$ ). The total decaheme cytochrome $c$ concentration per $\mathrm{OD}_{600}^{\mathrm{DR}}$, $[\mathrm{MtrC}+\mathrm{MtrA}]$, was then calculated by dividing the background corrected absorption at $552 \mathrm{~nm}$ normalized for $1 \mathrm{OD}_{600 \mathrm{~nm}}^{\mathrm{DR}}$ of cells by the effective path length of $0.08 \mathrm{~cm}$ and the extinction coefficient for MtrA and MtrC $\left.\left(\varepsilon_{552 \mathrm{~nm}}^{\mathrm{M} \text { trA,MtrC }}=280,000 \mathrm{M}^{-1} \mathrm{~cm}^{-1}\right)\right)^{30,57}$

Immunoblotting of $\mathrm{CcmE}$. The immunoblot of $\mathrm{C} \mathrm{cmE}$ was done similarly to Mavridou et al. ${ }^{58}$ The primary antibody, a rabbit antiserum to E. coli CcmE (gift from Stuart Ferguson) and the secondary antibody, goat anti-rabbit-peroxidase (Sigma, St. Louis, MO), were diluted $1 / 10,000$ and $1 / 20,000$, respectively. Development was performed using SuperSignal West Dura Substrate (Pierce, Rockford, IL). Blots were quantified using the ChemiDoc XRS+ System and software (Bio-Rad, Richmond, CA).

Electrochemistry. Cell pellets were resuspended in minimal M4 medium. An equivalent of $\mathrm{OD}_{600} \sim 0.04$ was inoculated into sterile bioreactors with $40 \mathrm{mM}$ L-lactate. Current was measured with a three-electrode electrochemical system with a working electrode maintained at $0.2 \mathrm{~V}$ versus $\mathrm{Ag} /$ $\mathrm{AgCl}$ (satd $\mathrm{KCl}$ ) using a potentiostat (VSP, BioLogic USA, Knoxville, TN). The working electrode was a $9 \mathrm{~cm}$ square piece of carbon cloth (PANEX 30-PW06, Zoltek Corp, St Louis, MO) attached to a graphite rod with carbon cement, and the counter electrode was a graphite rod. The bioreactors were maintained anaerobically by constant sparging with sterile $\mathrm{N}_{2}$ gas, ${ }^{54}$ stirred at $\sim 250 \mathrm{rpm}$ with magnetic stir bars, and maintained at $30{ }^{\circ} \mathrm{C} .{ }^{54}$ Lactate consumption was measured by HPLC at the end of the experiment.

\section{ASSOCIATED CONTENT}

\section{S Supporting Information}

Additional data showing the promoter calibrations, corrections and calibrations for diffuse reflectance, the predominance of MtrC and MtrA over endogenous cytochromes $c$, the 
correlation between the cell pellet color and diffuse reflectance, the linearity of $\mathrm{CcmE}$ immunoblotting data, a detailed description of the strains used, and more detailed methods description. This material is available free of charge via the Internet at http://pubs.acs.org.

\section{AUTHOR INFORMATION}

\section{Corresponding Author}

*Tel: 510-486-4299. Fax 510-495-2376. E-mail: cajo-franklin@ lbl.gov.

\section{Author Contributions}

C.P.G., H.M.J., M.A.T., N.B., Y.A., M.H., and C.A.-F. performed the experiments. G.C. and V.M. provided the pFAB\#-GFP constructs and guidance on $\mathrm{pFAB}$ promoters. L.T.A. and C.A.F. supervised the work. H.M.J. and C.A.-F. wrote the paper.

\section{Notes}

The authors declare no competing financial interest.

\section{ACKNOWLEDGMENTS}

We thank E. Chou and J. Bao for initial observations and B. Rad for helpful comments on the manuscript. We also thank Stuart Ferguson and Desponsia Mavridou for the generous gift of the $\mathrm{CcmE}$ antisera and advice on its use. Work performed at the Molecular Foundry was supported by the Office of Science, Office of Basic Energy Sciences, of the U.S. Department of Energy under Contract No. DE-AC02-05CH11231. C.P.G., N.B., Y.A. and C.A.-F. acknowledge support via the Molecular Foundry, and H.M.J. and M.B. acknowledge support from the Physical Biosciences Program, Office of Science, Office of Basic Energy Sciences, of the U.S. Department of Energy under Contract No. DE-AC02--05CH11231. M.A.T. and L.T.A. acknowledge support from the National Science Foundation through Career grant no. 0939882.

\section{ABBREVIATIONS}

IPTG, isopropyl $\beta$-D-1-thiogalactopyranoside; $\delta$-ALA, $\delta$-aminolevulinic acid; GFP, superfolding green fluorescent protein

\section{REFERENCES}

(1) Rabaey, K., and Rozendal, R. A. (2010) Microbial electrosynthesis - revisiting the electrical route for microbial production. Nat. Rev. Microbiol. 8, 706-716.

(2) Lovley, D. R., and Nevin, K. P. (2011) A shift in the current: new applications and concepts for microbe-electrode electron exchange. Curr. Opin. Biotechnol. 22, 441-449.

(3) Rosenbaum, M., and Angenent, L. T. (2010) Genetically modified microorganisms for bioelectrochemical systems, in Bioelectrochemical Systems: From Extracellular Electron Transfer to Biotechnological Application. (Rabaey, K., Angenent, L. T., Schröder, U., and Keller, J., Eds.), pp 101-117, International Water Association, London.

(4) Su, L., Jia, W., Hou, C., and Lei, Y. (2011) Microbial biosensors: a review. Biosens. Bioelectron. 26, 1788-1799.

(5) Benenson, Y. (2012) Biomolecular computing systems: principles, progress and potential. Nat. Rev. Genet. 13, 455-468.

(6) TerAvest, M. A., Li, Z., and Angenent, L. T. (2002) Bacteriabased biocomputing with Cellular Computing Circuits to sense, decide, signal, and act. Energy Environ. Sci. 4, 4907-4916.

(7) Qiao, Y., Li, C., Bao, S.-J., Lu, Z., and Hong, Y. (2008) Direct electrochemistry and electrocatalytic mechanism of evolved Escherichia coli cells in microbial fuel cells. Chem. Commun. (Cambridge, $U$. K.), 1290-1292.

(8) Alferov, S., Coman, V., Gustavsson, T., Reshetilov, A., von Wachenfeldt, C., Hägerhäll, C., and Gorton, L. (2009) Electrical communication of cytochrome enriched Escherichia coli JM109 cells with graphite electrodes. Electrochim. Acta 54, 4979-4984.

(9) Coman, V., Gustavsson, T., Finkelsteinas, A., von Wachenfeldt, C., Hägerhäll, C., and Gorton, L. (2009) Electrical wiring of live, metabolically enhanced Bacillus subtilis cells with flexible osmiumredox polymers. J. Am. Chem. Soc. 131, 16171-16176.

(10) Hasan, K., Patil, S., Górecki, K., Leech, D., Hägerhäll, C., and Gorton, L. (2012) Electrochemical communication between heterotrophically grown Rhodobacter capsulatus with electrodes mediated by an osmium redox polymer. Bioelectrochemistry, DOI: 10.1016/ j.bioelechem.2012.1005.1004.

(11) Jensen, H. M., Albers, A. E., Malley, K. R., Londer, Y. Y., Cohen, B. E., Helms, B. A., Weigele, P., Groves, J. T., and Ajo-Franklin, C. M. (2010) Engineering of a synthetic electron conduit in living cells. Proc. Natl. Acad. Sci. U.S.A. 107, 19213-19218.

(12) Kim, B. H., Kim, H. J., Hyun, M. S., and Park, D. H. (1999) Direct electrode reaction of Fe (III)-reducing bacterium, Shewanella putrefaciens. J. Microbiol. Biotechnol. 9, 127-131.

(13) Pinchuk, G., Rodionov, D., Yang, C., Li, X., Osterman, A., Dervyn, E., Geydebrekht, O., Reed, S., Romine, M., Collart, F., Scott, J., Fredrickson, J., and Beliaev, A. (2009) Genomic reconstruction of Shewanella oneidensis MR-1 metabolism reveals a previously uncharacterized machinery for lactate utilization. Proc. Natl. Acad. Sci. U.S.A. 106, 2874-2879.

(14) Brutinel, E., and Gralnick, J. (2012) Anomalies of the anaerobic tricarboxylic acid cycle in Shewanella oneidensis revealed by Tn-seq. Mol. Microbiol., DOI: 10.1111/j.1365-2958.2012.08196.x.

(15) Scott, J., and Nealson, K. (1994) A biochemical study of the intermediary carbon metabolism of Shewanella putrefaciens. J. Bacteriol. 176, 3408-3411.

(16) McMillan, D. G., Marritt, S., Butt, J., and Jeuken, L. (2012) Menaquinone-7 is specific cofactor in tetraheme quinol dehydrogenase CymA. J. Biol. Chem. 287, 14215-14225.

(17) Firer-Sherwood, M., Bewley, K., Mock, J.-Y., and Elliott, S. (2011) Tools for resolving complexity in the electron transfer networks of multiheme cytochromes c. Metallomics 3, 344-348.

(18) Myers, C., and Myers, J. (1997) Cloning and sequence of cymA, a gene encoding a tetraheme cytochrome $c$ required for reduction of iron(III), fumarate, and nitrate by Shewanella putrefaciens MR-1. J. Bacteriol. 179, 1143-1152.

(19) Bretschger, O., Obraztsova, A., Sturm, C., Chang, I., Gorby, Y., Reed, S., Culley, D., Reardon, C., Barua, S., Romine, M., Zhou, J., Beliaev, A., Bouhenni, R., Saffarini, D., Mansfeld, F., Kim, B.-H., Fredrickson, J., and Nealson, K. (2007) Current production and metal oxide reduction by Shewanella oneidensis MR-1 wild type and mutants. Appl. Environ. Microbiol. 73, 7003-7012.

(20) Hartshorne, R., Reardon, C., Ross, D., Nuester, J., Clarke, T., Gates, A., Mills, P., Fredrickson, J., Zachara, J., Shi, L., Beliaev, A., Marshall, M., Tien, M., Brantley, S., Butt, J., and Richardson, D. (2009) Characterization of an electron conduit between bacteria and the extracellular environment. Proc. Natl. Acad. Sci. U.S.A. 106, 2216922174.

(21) Ross, D., Ruebush, S., Brantley, S., Hartshorne, R., Clarke, T., Richardson, D., and Tien, M. (2007) Characterization of proteinprotein interactions involved in iron reduction by Shewanella oneidensis MR-1. Appl. Environ. Microbiol. 73, 5797-5808.

(22) Brutinel, E., and Gralnick, J. (2012) Shuttling happens: soluble flavin mediators of extracellular electron transfer in Shewanella. Appl. Microbiol. Biotechnol. 93, 41-48.

(23) Clarke, T., Edwards, M., Gates, A., Hall, A., White, G., Bradley, J., Reardon, C., Shi, L., Beliaev, A., Marshall, M., Wang, Z., Watmough, N., Fredrickson, J., Zachara, J., Butt, J., and Richardson, D. (2011) Structure of a bacterial cell surface decaheme electron conduit. Proc. Natl. Acad. Sci. U.S.A. 108, 9384-9389.

(24) Coursolle, D., Baron, D., Bond, D., and Gralnick, J. (2010) The Mtr respiratory pathway is essential for reducing flavins and electrodes in Shewanella oneidensis. J. Bacteriol. 192, 467-474.

(25) Marsili, E., Baron, D., Shikhare, I., Coursolle, D., Gralnick, J., and Bond, D. (2008) Shewanella secretes flavins that mediate 
extracellular electron transfer. Proc. Natl. Acad. Sci. U.S.A. 105, 39683973.

(26) von Canstein, H., Ogawa, J., Shimizu, S., and Lloyd, J. (2008) Secretion of flavins by Shewanella species and their role in extracellular electron transfer. Appl. Environ. Microbiol. 74, 615-623.

(27) Jiang, X., Hu, J., Fitzgerald, L., Biffinger, J., Xie, P., Ringeisen, B., and Lieber, C. (2010) Probing electron transfer mechanisms in Shewanella oneidensis MR-1 using a nanoelectrode platform and singlecell imaging. Proc. Natl. Acad. Sci. U.S.A. 107, 16806-16810.

(28) Richardson, D., Butt, J., Fredrickson, J., Zachara, J., Shi, L., Edwards, M., White, G., Baiden, N., Gates, A., Marritt, S., and Clarke, T. (2012) The 'porin-cytochrome' model for microbe-to-mineral electron transfer. Mol. Microbiol. 85, 201-212.

(29) Ross, D. E., Flynn, J. M., Baron, D. B., Gralnick, J. A., and Bond, D. R. (2011) Towards electrosynthesis in Shewanella: energetics of reversing the Mtr pathway for reductive metabolism. PLoS One 6, 9.

(30) Pitts, K., Dobbin, P., Reyes-Ramirez, F., Thomson, A., Richardson, D., and Seward, H. (2003) Characterization of the Shewanella oneidensis MR-1 decaheme cytochrome MtrA: expression in Escherichia coli confers the ability to reduce soluble Fe(III) chelates. J. Biol. Chem. 278, 27758-27765.

(31) Myers, C., and Myers, J. (2003) Cell surface exposure of the outer membrane cytochromes of Shewanella oneidensis MR-1. Lett. Appl. Microbiol. 37, 254-258.

(32) Beliaev, A., Saffarini, D., McLaughlin, J., and Hunnicut, D. (2001) MtrC, an outer membrane decahaem $c$ cytochrome required for metal reduction in Shewanella putrefaciens MR-1. Mol. Microbiol. 39, $722-730$.

(33) Myers, C., and Myers, J. (2002) MtrB is required for proper incorporation of the cytochromes OmcA and OmcB into the outer membrane of Shewanella putrefaciens MR-1. Appl. Environ. Microbiol. 68, 5585-5594.

(34) Bagos, P., Liakopoulos, T., Spyropoulos, I., and Hamodrakas, S. (2004) PRED-TMBB: a web server for predicting the topology of beta-barrel outer membrane proteins. Nucleic Acids Res. 32, 4.

(35) Schicklberger, M., Bucking, C., Schuetz, B., Heide, H., and Gescher, J. (2011) Involvement of the Shewanella oneidensis decaheme cytochrome MtrA in the periplasmic stability of the beta-barrel protein MtrB. Appl. Environ. Microbiol. 77, 1520-1523.

(36) Grove, J., Tanapongpipat, S., Thomas, G., Griffiths, L., Crooke, H., and Cole, J. (1996) Escherichia coli K-12 genes essential for the synthesis of c-type cytochromes and a third nitrate reductase located in the periplasm. Mol. Microbiol. 19, 467-481.

(37) Arslan, E., Schulz, H., Zufferey, R., Kunzler, P., and ThonyMeyer, L. (1998) Overproduction of the Bradyrhizobium japonicum ctype cytochrome subunits of the $\operatorname{cbb}(3)$ oxidase in Escherichia coli. Biochem. Biophys. Res. Commun. 251, 744-747.

(38) Thony-Meyer, L., Kunzler, P., and Hennecke, H. (1996) Requirements for maturation of Bradyrhizobium japonicum cytochrome c(550) in Escherichia coli. Eur. J. Biochem. 235, 754-761.

(39) Sanders, C., Turkarslan, S., Lee, D.-W., and Daldal, F. (2010) Cytochrome $c$ biogenesis: the Ccm system. Trends Microbiol. 18, 266274.

(40) Donald, J., Hicks, M., Richardson, D., and Palmer, T. (2008) The c-type cytochrome OmcA localizes to the outer membrane upon heterologous expression in Escherichia coli. J. Bacteriol. 190, 51275131 .

(41) Wagner, S., Baars, L., Ytterberg, A. J., Klussmeier, A., Wagner, C. S., Nord, O., Nygren, P. A., van Wijk, K. J., and de Gier, J. W. (2007) Consequences of membrane protein overexpression in Escherichia coli. Mol. Cell. Proteomics 6, 1527-1550.

(42) Wickström, D., Wagner, S., Baars, L., Ytterberg, A., Klepsch, M., van Wijk, K., Luirink, J., and de Gier, J.-W. (2011) Consequences of depletion of the signal recognition particle in Escherichia coli. J. Biol. Chem. 286, 4598-4609.

(43) Gao, T., and O'Brian, M. R. (2007) Control of DegP-dependent degradation of c-type cytochromes by heme and the cytochrome $c$ maturation system in Escherichia coli. J. Bacteriol. 189, 6253-6259.
(44) Fernandes, A. P., Couto, I., Morgado, L., Londer, Y. Y., and Salgueiro, C. A. (2008) Isotopic labeling of c-type multiheme cytochromes overexpressed in E. coli. Protein Expression Purif. 59, $182-188$.

(45) Londer, Y. Y. (2011) Expression of recombinant cytochromes $c$ in E. coli. Methods Mol. Biol. 705, 123-150.

(46) Londer, Y., Pokkuluri, P., Erickson, J., Orshonsky, V., and Schiffer, M. (2005) Heterologous expression of hexaheme fragments of a multidomain cytochrome from Geobacter sulfurreducens representing a novel class of cytochromes c. Protein Expression Purif. $39,254-260$.

(47) Londer, Y. Y., Pokkuluri, P. R., Orshonsky, V., Orshonsky, L., and Schiffer, M. (2006) Heterologous expression of dodecaheme 'nanowire' cytochromes $c$ from Geobacter sulfurreducens. Protein Expression Purif. 47, 241-248.

(48) Gescher, J., Cordova, C., and Spormann, A. (2008) Dissimilatory iron reduction in Escherichia coli: identification of CymA of Shewanella oneidensis and NapC of E. coli as ferric reductases. Mol. Microbiol. 68, 706-725.

(49) Narayanan, A., Ridilla, M., and Yernool, D. (2011) Restrained expression, a method to overproduce toxic membrane proteins by exploiting operator-repressor interactions. Protein Sci. 20, 51-61.

(50) Wagner, S., Klepsch, M. M., Schlegel, S., Appel, A., Draheim, R., Tarry, M., Hogbom, M., van Wijk, K. J., Slotboom, D. J., Persson, J. O., and de Gier, J. W. (2008) Tuning Escherichia coli for membrane protein overexpression. Proc. Natl. Acad. Sci. U.S.A. 105, 14371-14376.

(51) Kelly, J. R., Rubin, A. J., Davis, J. H., Ajo-Franklin, C. M., Cumbers, J., Czar, M. J., de Mora, K., Glieberman, A. L., Monie, D. D., and Endy, D. (2009) Measuring the activity of BioBrick promoters using an in vivo reference standard. J. Biol. Eng. 3, 4.

(52) Engler, C., Gruetzner, R., Kandzia, R., and Marillonnet, S. (2009) Golden gate shuffling: a one-pot DNA shuffling method based on type IIs restriction enzymes. PLoS One, 4.

(53) Scott, M., Gunderson, C., Mateescu, E., Zhang, Z., and Hwa, T. (2010) Interdependence of cell growth and gene expression: origins and consequences. Science (New York, N.Y.) 330, 1099-1102.

(54) Venkataraman, A., Rosenbaum, M. A., Perkins, S. D., Werner, J. J., and Angenent, L. T. (2011) Metabolite-based mutualism between Pseudomonas aeruginosa PA14 and Enterobacter aerogenes enhances current generation in bioelectrochemical systems. Energy Environ. Sci. 4, 4550-4559.

(55) McGuirl, M., Lee, J., Lyubovitsky, J., Thanyakoop, C., Richards, J., Gray, H., and Winkler, J. (2003) Cloning, heterologous expression, and characterization of recombinant class II cytochromes $c$ from Rhodopseudomonas palustris. Biochim. Biophys. Acta 1619, 23-28.

(56) Xiong, Y., Chen, B., Shi, L., Fredrickson, J., Bigelow, D., and Squier, T. (2011) Targeted protein degradation of outer membrane decaheme cytochrome MtrC metal reductase in Shewanella oneidensis MR-1 measured using biarsenical probe CrAsH-EDT(2). Biochemistry 50, 9738-9751.

(57) Hartshorne, R., Jepson, B., Clarke, T., Field, S., Fredrickson, J., Zachara, J., Shi, L., Butt, J., and Richardson, D. (2007) Characterization of Shewanella oneidensis MtrC: a cell-surface decaheme cytochrome involved in respiratory electron transport to extracellular electron acceptors. J. Biol. Inorg. Chem. 12, 1083-1094.

(58) Mavridou, D. A. I., Stevens, J. M., Mönkemeyer, L., Daltrop, O., di Gleria, K., Kessler, B. M., Ferguson, S. J., and Allen, J. W. A. (2012) A pivotal heme-transfer reaction intermediate in cytochrome $c$ biogenesis. J. Biol. Chem. 287, 2342-2352. 